\title{
Palladium Nanoparticles within Core-Cross-Linked Polymer Gels for Suzuki Coupling Reactions: from Monomers to Ready-to-Use Catalysts in Two-Steps
}

\author{
Tanize Bortolotto, ${ }^{a}$ Gabrielly E. Neumann, ${ }^{a}$ Suelen G. Trindade, ${ }^{a}$ Laura C. E. da Silva, ${ }^{b}$ \\ Oscar E. D. Rodrigues, ${ }^{a}$ Vanessa Schmidt ${ }^{a}$ and Cristiano Giacomelli*,a \\ ${ }^{a}$ Departamento de Química, Universidade Federal de Santa Maria, \\ 97105-900 Santa Maria-RS, Brazil \\ ${ }^{b}$ Instituto de Química, Universidade Estadual de Campinas, \\ C. P. 6154, 13083-970 Campinas-SP, Brazil
}

\begin{abstract}
This study shows that polymer nanogels (well-defined cross-linked nanoparticles behaving as highly hydrated unimolecular objects) are suitable capping agents for palladium nanoparticle catalyst systems. In spite of the structurally complex nature of the catalysts, it was possible to go from acrylate-based monomers to ready-to-use and recyclable catalysts in only two-steps. First, nanogels were synthesized by activators regenerated by electron transfer atom transfer radical polymerization (ARGET ATRP) method with sequential addition of monomers. Then, in the second step, palladium acetate was reduced to obtain ready-to-use catalysts (ca. $25 \mathrm{~nm}$ nanogel structures containing ca. $7 \mathrm{~nm}$ palladium nanoparticles). The catalytic activity was confirmed in $p$-nitrophenol (Nip) reduction and Suzuki cross-coupling reactions. The observed rate constants $\left(\mathrm{k}_{\mathrm{obs}}\right)$ for Nip reduction were in the range of $0.8-10.0 \times 10^{-2} \mathrm{~s}^{-1}$ depending on the polymeric capping agent structure, with the highest value being found for nanogel-based systems. These composite catalysts also mediated the cross-coupling Suzuki reaction providing the expected products in quantitative yields under relatively mild conditions after $4 \mathrm{~h}$ at $50{ }^{\circ} \mathrm{C}$. The simplicity of catalyst preparation protocol that ensures excellent activity and the low concentration of polymer applied during the synthesis are a step forward in terms of environmental and economic prospects.
\end{abstract}

Keywords: polymers, capping agents, palladium nanoparticles, $p$-nitrophenol reduction, Suzuki-Miyaura reaction

\section{Introduction}

Carbon-carbon couplings through Suzuki, Sonogashira and Heck reactions are ubiquitous in organic chemistry due to their convenience in producing various chemicals in many industry fields. ${ }^{1}$ These reactions are usually carried out in presence of metallic species that work as homogeneous and/or heterogeneous catalysts. Currently, the better product quality and reduced environmental impact (simple protocols for catalyst recovery and fewer purification steps) attract most of the attention to heterogeneous systems. ${ }^{1}$

In this context, palladium nanoparticles (PdNPs) are proven efficient heterogeneous catalysts, although homogenous and heterogeneous mechanisms, rather than being mutually exclusive, should be considered as

*e-mail: cgiacomelli@ufsm.br complementary and dependent on the immobilization degree of PdNPs, as noted by Pérez-Lorenzo. ${ }^{2}$

The usually high activity of PdNPs is attributed to electronic properties and under-coordination of surface atoms, which are sensitive to particle size and shape, and inter-particle distance and interaction mechanisms. ${ }^{3}$ The high surface energy also renders PdNPs systems prone to aggregation and loss of activity ${ }^{3-5}$ whenever the surface chemistry is not properly controlled. As a consequence, a great deal of effort has been put into the development of simple and effective strategies allowing for precise engineering of surface properties. In fact, most of the solutions make use of capping agents such as surfactants, peptides, ionic liquids, dendrimers or polymers, ${ }^{1}$ which ultimately define the catalytic activity and substrate selectivity of PdNP catalysts. ${ }^{1,3}$ Therefore, the use of any capping agent obviously transforms the behavior of palladium sols. 
Lately, the use of smart polymer coatings that bind rather weakly to PdNPs surface through heteroatoms (electron donors) playing the role of ligands have attracted much attention, ${ }^{6}$ essentially because the degree of sophistication is only limited by the creativity of the polymer chemist. ${ }^{7}$ A myriad of polymer capping agents can be designed to react quite specifically depending on the environmental surroundings. ${ }^{8}$

In a recent work on the same topic as herein discussed, we showed that high catalytic activity is obtained whenever the catalyst features a less compact, more hydrated and consequently more extended polymer chain conformation. ${ }^{9}$ Functional polymers and block copolymers that interact with palladium nanoparticles mainly through tertiary nitrogen atoms are suitable capping agents and can be used in relatively small amounts. As a consequence, catalysts with large active surface area and effective mass transport of reactants and products across the stabilizing layer are obtained.

These findings correlate well with studies carried out by others. Chen et al., ${ }^{10}$ who used poly(ethylene glycol)-co-poly( $N$-isopropylacrylamide) to synthesize and stabilize sub-nanometer Pd clusters, found that the catalyst showed excellent activity because the environment around the hybrid organic-inorganic catalyst was enriched with both hydrophilic and hydrophobic reactants (pre-concentration effect). The use of polymers modified with ligands that interact specifically with palladium species was explored very recently and successfully. Fan et al. ${ }^{11}$ developed a catalyst system consisting of palladium nanoparticles supported on cross-linked polyaniline with bulky phosphorus-based ligands. An impressively simple and yet highly efficient approach was invented by Chen et al., ${ }^{12}$ who took advantage of a very cheap polymer synthesis method followed by post-polymerization functionalization with bis(imino) pyridine ligands that formed a coordination complex with palladium anchored to the polymer matrix.

It then became clear to us that, as an evolution from the earlier study, ${ }^{9}$ core cross-linked star (CCS) polymers could congregate all of the advantages described above. Consisting of well-defined cross-linked nanoparticles with a water-soluble polymer at the outer shell and an either compact or swollen core, they behave as highly hydrated unimolecular single objects ${ }^{13-16}$ (so-called nanogels) through which reactants and products of organic reactions can diffuse without big constrains. Indeed, Chen et al..$^{14}$ and Zhang et al. ${ }^{13}$ formerly pointed out the use of CCS polymers as interfacial stabilizers, which affect colloidal systems to an extent intermediate between soluble polymers and colloidal particles. Previous studies by Seto et al..$^{17,18}$ showed that the catalytic activity of palladium nanoparticles loaded in nanogels of amino-functionalized polymers was higher than that of bulk palladium, and that such systems can be used to devise continuous-flow Suzuki coupling reactors. Recently, da Costa et al..$^{19}$ also noted that core-shell nanogels are attractive stabilizers and supports for catalytically active metallic nanoparticles by functioning as palladium reservoirs wherein the reaction takes place in a quasi-homogeneous fashion.

In this study, we describe the preparation and application of core cross-linked star (CCS) polymers (nanogels) as stabilizers and supports $\mathrm{Pd}^{0}$ nanoparticles. Nanogels were synthesized by the straightforward, easy to perform, and environmentally friendly activators regenerated by electron transfer atom transfer radical polymerization (ARGET ATRP) method. ${ }^{20,21}$ The catalytic activity of polymer@PdNPs was evaluated comparatively using a model reaction ( $p$-nitrophenol reduction) and typical Suzuki cross-coupling reactions. The results were interpreted in the light of macromolecular composition and architecture, and metal nanoparticle size and distribution.

\section{Experimental}

\section{Materials}

Poly(ethylene glycol) methyl ether methacrylate (POEGMA, Aldrich, two samples, being one of average $\mathrm{M}_{\mathrm{n}}$ (number-average molar mass) $=500 \mathrm{~g} \mathrm{~mol}^{-1}$, and the other of average $M_{n}=300 \mathrm{~g} \mathrm{~mol}^{-1}$ ), 2-(diethylamino)ethyl methacrylate (DEA, Aldrich, 99\%), divinylbenzene (DVB, Aldrich, 80\%), ethylene glycol dimethacrylate (EGDMA, Aldrich, 98\%) were purified by passing over a column of neutral alumina prior to use. Palladium acetate (Aldrich, $>99.9 \%$ ), sodium borohydride (Aldrich, > 96\%), ethyl $\alpha$-bromoisobutyrate (EBiB, Aldrich, 98\%), copper(II) bromide $\left(\mathrm{CuBr}_{2}\right.$, Aldrich, 99\%), tin(II) 2-ethylhexanoate $\left(\mathrm{Sn}(\mathrm{EH})_{2}\right.$, Aldrich, 92.5-100\%), tris(2-pyridylmethyl) amine (TPMA, Aldrich, 98\%), L-ascorbic acid (Aldrich, 99.7-100.5\%), methanol (Aldrich, 99.8\%), deuterated chloroform $\left(\mathrm{CDCl}_{3}\right)$, and hexane (Vetec, $\left.95 \%\right)$ were used as received. Glassware was cleaned with aqua regia and rinsed thoroughly with deionized water. Pure water was collected from a Millipore Alpha Q system (conductivity $<0.05 \mu \mathrm{S} \mathrm{cm}^{-1}$ ) and used to prepare aqueous solutions.

\section{Instrumentation}

\section{Gel permeation chromatography (GPC)}

$M_{n}$ and dispersity $\left(M_{w} / M_{n}\right)$ values were determined by GPC in tetrahydrofuran (THF) at a flow rate of $1.0 \mathrm{~mL} \mathrm{~min}^{-1}$ 
using SKgel $\mathrm{H}_{\mathrm{XL}}$ guard and TSKgel $\mathrm{G} 3000 \mathrm{H}_{\mathrm{XL}}$ columns thermostated at $40{ }^{\circ} \mathrm{C}$ on a Shimadzu apparatus equipped with an SCK-10A controller, DGU-20A degassing unit, LC-10AD solvent delivery module, CTO-20A column oven, and RID-10A refractive index and SPD-20A UV-Vis detectors. Calibration was performed using a series of near-monodisperse polystyrene (PS) standards.

\section{Dynamic light scattering (DLS)}

DLS measurements were performed using a Brookhaven laser light scattering system consisting of a $75 \mathrm{~mW}$ He-Ne linear polarized laser source operating at a wavelength $(\lambda)$ of $632.8 \mathrm{~nm}$, a BI-200SM laser goniometer and a BI-APD avalanche photodiode detector. The autocorrelation functions were acquired using a BI-9000AT digital correlator, and recorded using the BI-DLSW Correlator Control software. Samples were placed in $10 \mathrm{~mm}$ diameter glass cells and maintained at a constant temperature of $25.0 \pm 0.1{ }^{\circ} \mathrm{C}$. The accessible scattering angles ranged from $15^{\circ}$ to $150^{\circ}$. The measured intensity correlation functions $\mathrm{g}_{2}(\mathrm{t})$ were determined using the algorithm REPES (incorporated in the GENDIST program). The hydrodynamic radius $\left(\mathrm{R}_{\mathrm{H}}\right)$ (or diameter, $D=2 R_{H}$ ) was calculated from the Stokes-Einstein relation, and polydispersity, where indicated, was estimated from the analysis of cumulants.

\section{UV-Vis spectroscopy}

UV-Vis spectra were recorded using a Shimadzu UV2600 spectrophotometer. For the measurements, $3.0 \mathrm{~mL}$ of solution were placed in a $10 \mathrm{~mm}$ square quartz cell. All spectra were recorded in the wavelength range of 200-800 $\mathrm{nm}$ at a scan rate of $600 \mathrm{~nm} \mathrm{~min}^{-1}(0.1 \mathrm{~s}$ integration per $1.0 \mathrm{~nm}$ ) for air-equilibrated thermostated solutions under stirring.

\section{Transmission electron microscopy (TEM)}

Nanoparticles morphology, size and size distribution were investigated in a Carl Zeiss Libra 120 Plus transmission electron microscope equipped with an in-column OMEGA filter, using a tungsten thermionic source at an acceleration voltage of $80 \mathrm{kV}$. Samples were prepared by depositing a drop of $0.1 \mathrm{mg} \mathrm{mL}^{-1}$ nanoparticle dispersion on a carbon coated copper grid. The droplet was left to settle for $1 \mathrm{~min}$ and gently dried using filter paper. Nanoparticle average size was obtained from a sample of at least 300 nanoparticles using Image $\mathrm{J} \circledast$ software.

\section{Nuclear magnetic resonance (NMR)}

${ }^{1} \mathrm{H}$ NMR (400 MHz) spectra were acquired using an Avance DPX 400 spectrometer with $\mathrm{D}_{2} \mathrm{O}$, DMSO- $d_{6}$ or $\mathrm{CDCl}_{3}$ as the solvent, as indicated.

\section{General procedure for CCS polymer (nanogel) synthesis}

The polymer samples used in this study are listed in Table 1. Linear homopolymers (1-2) and linear diblock copolymers (3-4) were considered in this investigation in order to clarify the relationship between macromolecular architecture (linear homopolymer, linear diblock copolymer and CCS copolymers) and catalytic activity of PdNPs. Sample $\mathbf{1}$ is commercially available, and sample $\mathbf{3}$ was synthesized following the procedures described by Liu et al. ${ }^{22}$ with adaptations, as described in detail elsewhere. ${ }^{23}$

Table 1. Molecular characteristics of polymers used in this study

\begin{tabular}{|c|c|}
\hline entry & $\begin{array}{l}\text { Polymer }^{\mathrm{a}} \\
\text { Highlights of the sample }\end{array}$ \\
\hline 1 & $\begin{array}{l}\mathrm{PEO}_{113} \\
\text { linear homopolymer; commonly used in PdNP Suzuki reactions }\end{array}$ \\
\hline 2 & $\begin{array}{c}\text { PPOEGMA }_{67} \\
\text { bottlebrush polymer; with methacrylate backbone and short } \\
\text { water-soluble side chains made of ethylene oxide units }\end{array}$ \\
\hline 3 & $\begin{array}{l}\qquad \mathrm{PEO}_{113}-b \text {-PDEA } \\
\text { linear diblock copolymer; forms core-shell micelles in water; } \\
\text { micelle core interacts with metals via nitrogen atom (electron } \\
\text { donor) }\end{array}$ \\
\hline 4 & $\begin{array}{l}\text { PPOEGMA }_{45}-b \text {-PDEA } \\
\text { linear diblock copolymer; bottlebrush-block-linear polymer } \\
\text { forming core-shell micelles in water; the micelle core is the same } \\
\text { as in 3, but the shell is made of shorter bottlebrush-like chains }\end{array}$ \\
\hline 5 & $\begin{array}{l}\text { PPOEGMA }_{60}-b-\mathrm{P}\left(\mathrm{POEGMA}-\mathrm{co}-\mathrm{EGDMA}_{2}\right) \\
\text { core cross-linked star polymer; arms of consist of bottlebrushes; } \\
\text { absence of electron donor nitrogen atoms that interact with } \\
\text { palladium }\end{array}$ \\
\hline 6 & $\begin{array}{l}\text { PPOEGMA }_{60}-b \text {-P(POEGMA-co-EGDMA-co-DEA) } \\
\text { core cross-linked star polymer; similar to } 5 \text { but the core contains } \\
\text { nitrogen atoms capable of interaction with palladium without } \\
\text { poisoning }\end{array}$ \\
\hline
\end{tabular}

${ }^{\text {a Subscripts refer to the targeted mean degree of polymerization (DP) of }}$ each block. PEO: poly(ethylene oxide); PPOEGMA: poly[poly(ethylene glycol) methyl ether methacrylate]; PDEA: poly[2-(diethylamino) ethyl methacrylate]; POEGMA: poly(ethylene glycol) methyl ether methacrylate; EGDMA: ethylene glycol dimethacrylate; DEA: 2-(diethylamino)ethyl methacrylate.

Linear homopolymer $\mathbf{2}$, linear diblock copolymer $\mathbf{4}$ and nanogels 5-6 were synthesized employing a convenient onepot two-step method based on ARGET ATRP polymerization technique, following a protocol adapted from earlier communications by Matyjaszewski and co-workers ${ }^{24-26}$ on the topic. In a typical procedure, methanol as solvent

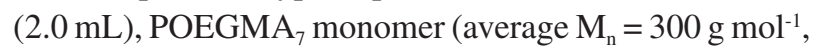
$3.15 \mathrm{~g}, 10.5 \mathrm{mmol}), \mathrm{CuBr}_{2}(0.34 \mathrm{mg}, 0.0015 \mathrm{mmol})$, TPMA ligand (2.60 mg, $0.0090 \mathrm{mmol})$, EBiB initiator $(29.3 \mathrm{mg}$, $0.15 \mathrm{mmol}), \mathrm{Sn}(\mathrm{EH})_{2}$ reducing agent $(12.2 \mathrm{mg}, 0.03 \mathrm{mmol})$ and dimethylformamide as internal reference for NMR spectroscopy conversion analysis $(300 \mu \mathrm{L})$ were loaded 
into a dry $50 \mathrm{~mL}$ Schlenk tube previously purged with $\mathrm{N}_{2}$. The mixture in the tube was subjected to gentle purge with $\mathrm{N}_{2}$ for $30 \mathrm{~min}$. Then, the Schlenk tube was closed, and immediately immersed in an oil bath at $90{ }^{\circ} \mathrm{C}$ to start the first step of the polymerization, unless otherwise indicated. Samples were taken periodically with an airtight degassed syringe for conversion analysis, wherever indicated. The polymerization was stopped by cooling down to room temperature and opening the flask to air when the conversion of $\mathrm{POEGMA}_{7}$ monomer reached $>90 \%$.

Reactants for the second reaction step were then added to the same Schlenk flask. Degassed EGDMA as bifunctional cross-linker monomer ( $89 \mathrm{mg}, 0.450 \mathrm{mmol})$, DEA as spacing and amino-functional monomer $(1.38 \mathrm{~g}$, $7.5 \mathrm{mmol})$ and methanol $(1.5 \mathrm{~mL})$ were charged into the flask, which was then closed and immersed in an oil bath at $70{ }^{\circ} \mathrm{C}$ to continue the polymerization for ca. $2 \mathrm{~h}$, and thus generate the CCS polymer (nanogel). The final product was obtained after precipitation in cold ethyl ether and vacuum drying for at least $48 \mathrm{~h}$. Table 1 summarizes the macromolecular characteristics of all samples used in this study.

\section{Nanoparticle synthesis}

PdNP synthesis in the presence of $\mathrm{pH}$-responsive polymers was carried out by mixing $2.48 \mathrm{~mL}$ of $0.1 \mathrm{~mol} \mathrm{~L}^{-1}$ buffer solution (phosphates at $\mathrm{pH} 7.4$, or sodium tetraborate at $\mathrm{pH} 9.1$ ) with $2.48 \mathrm{~mL}$ of $\mathrm{pH}$ ca. 3.5 aqueous solutions containing molecularly dissolved polymer chains $(5.0 \mathrm{mg})$ and $\mathrm{Pd}\left(\mathrm{CH}_{3} \mathrm{COO}\right)_{2}\left(3.09 \times 10^{-5} \mathrm{~g} ; 1.38 \times 10^{-3} \mathrm{mmol}\right)$. Soon after, $\mathrm{NaBH}_{4}(42 \mu \mathrm{L}$ of $0.10 \mathrm{~mol} \mathrm{~L}-1,0.001 \mathrm{~g}$; $4.10 \times 10^{-3} \mathrm{mmol}$ ) was added to the mixture and the solution turned dark gray. The final volume of the solution was $5.0 \mathrm{~mL}$. In the case of non-responsive polymers (those without secondary amino groups), the synthesis was carried out through reactions with the same stoichiometry, but the polymer and palladium salt were directly dissolved in water or buffer solution prior to the addition of the reducing agent. The nanoparticle composition is referred to as polymer@PdNP (e.g.1@PdNP) throughout the text.

\section{Catalytic activity assays}

\section{Reduction of $p$-nitrophenol (Nip)}

The catalytic activity of the polymer-coated PdNPs was first evaluated for the reduction of $p$-nitrophenol (Nip) to $p$-aminophenol (Amp) using UV-Vis spectroscopy. ${ }^{27-29}$ The reaction was carried out in a quartz cell using an aqueous solution $(2.5 \mathrm{~mL})$ containing Nip $\left(3.00 \times 10^{-4} \mathrm{mmol}\right)$ and $\mathrm{NaOH}\left(3.00 \times 10^{-3} \mathrm{mmol}\right)$. After the addition of the reducing agent $\left(1.50 \times 10^{-2} \mathrm{mmol} \mathrm{NaBH}_{4}\right)$, the cell was placed into the cell holder and the solution was kept under stirring. Finally, a solution containing PdNP $\left(7.37 \times 10^{-5} \mathrm{mmol}\right.$ of Pd) was added, and the reaction was monitored through the decrease in the absorbance of the $p$-nitrophenolate ions at $400 \mathrm{~nm} \cdot$. $^{27,28}$

\section{Suzuki cross-coupling reactions}

Suzuki coupling reactions were carried out using PdNPs coated with different macromolecules. In a typical procedure, $\mathrm{K}_{2} \mathrm{CO}_{3}(0.5 \mathrm{mmol})$, phenylboronic acid $(0.375 \mathrm{mmol})$ and polymer-coated PdNPs $(0.025 \mathrm{mmol}$ of Pd; $10 \mathrm{~mol} \%$, unless otherwise indicated) were added to $1.6 \mathrm{~mL}$ of a $50 \% \mathrm{v} / \mathrm{v}$ ethyl alcohol/water solution. Next, 4-iodoanisole $(0.25 \mathrm{mmol})$ was added to the solution, the tube was sealed and the reaction was kept under stirring at $50{ }^{\circ} \mathrm{C}$. After $4 \mathrm{~h}$, the reaction mixture was cooled down to room temperature and filtered under vacuum using a Teflon membrane (TE36 $0.45 \mu \mathrm{m}, 47 \mathrm{~mm}$ ). The solvent was removed by rotatory evaporation and the final product was purified by column chromatography using silica gel as the stationary phase and ethyl acetate/hexane (5/95) as the eluent. The reaction progress was monitored by thin layer chromatography and ${ }^{1} \mathrm{H}$ NMR. The isolated product was characterized by ${ }^{1} \mathrm{H}$ NMR $\left(\left(\mathrm{CDCl}_{3}, 200 \mathrm{MHz}, 298 \mathrm{~K}\right) \delta\right.$ (ppm) 7.49-6.88 (9H, m, Ph), $\left.3.77\left(3 \mathrm{H}, \mathrm{s}, \mathrm{OCH}_{3}\right)\right)$ and mass spectrometry $(\mathrm{m} / \mathrm{z}$ calcd. for $\mathrm{C}_{13} \mathrm{H}_{12} \mathrm{O}[\mathrm{M}]^{+}$184.1; found 184.1).

\section{Results and Discussion}

\section{PdNP catalyst synthesis}

The main premise that formed the basis of this study was keeping the catalyst synthesis protocol easy to perform and environmentally friendly. We therefore selected a set of monomers that could lead to polymers capable of interacting with PdNP without poisoning catalytic sites. Besides, capping agents would have to assemble onto the PdNP surface to generate a permeable (non-compact) stabilizing layer through which reactants and products would diffuse with minimal constrains. The choice of monomers was restricted to those showing considerable solubility in water.

Based on previous studies, ${ }^{9}$ DEA and POEGMA were considered suitable monomers for this work. Whilst the former is $\mathrm{pH}$-responsive and its typical $\mathrm{p} K_{\mathrm{a}}$ implies in partial protonation at near neutral $\mathrm{pH}$ solutions, the latter is a water-soluble bottlebrush. Both of these monomers can be polymerized either in bulk or in methanol by the ARGET ATRP method. This polymerization approach is particularly attractive because it uses very low copper concentrations, 
it does not require stringent experimental conditions (it is, in fact, tolerant to reactants impurities and can even be performed in air), and it is suited for industrial scale up.

The polymer synthesis strategy developed in this study is shown in Scheme 1 for a representative sample. In all cases, the first reaction (i) (top left part of Scheme 1) consisted in the preparation of a linear homopolymer via a reaction that was carried out up to conversions $>90 \%$. Under these circumstances, a high degree of bromine chain-end functionality is ensured by ARGET ATRP. ${ }^{21,30-32}$ Thus, the resulting polymers could be chain-extended subsequently in reaction (ii) (top right part of Scheme 1) with either a monovinyl or a divinyl monomer, leading to the corresponding block copolymers or CCS polymers, respectively. The reaction pathway is similar to that employed by Cao et al. ${ }^{16}$ The CCS polymers, in particular, are formed by cross-linking of preformed macroinitiators via polymerization in a convergent fashion (arm-first star polymer synthesis method). Although the CCS macromolecule is structurally complex, the synthetic method is really simple. After cooling down the reaction mixture of the first step, reactants for the second step were added directly into the same flask (sequential monomer addition technique). Hence, all products were obtained in a one-pot two-step approach employing commercially available reactants.

In the sequence, PdNPs were synthesized using $\mathrm{NaBH}_{4}$ as the reducing agent and the polymers listed in Table 1 as stabilizers. The set of samples included linear homopolymers (1-2), linear diblock copolymers (3-4) and
CCS polymers (5-6). As given in the highlights of each sample in Table 1, the macromolecular characteristics became more complex going from $\mathbf{1}$ to $\mathbf{6}$. The overall effect of the polymer architecture is discussed below along with results from catalytic activity assays.

In order to ensure that the reduction of $\mathrm{Pd}^{\mathrm{II}}$ ions to $\mathrm{Pd}^{0}$ was similar for all of the systems regardless of the nature of the polymer stabilizer, the reaction was conducted in the presence of $\mathrm{NaBH}_{4}$. Most of the polymers listed in Table 1 can, in fact, work both as reducing agents and catalyst supports. However, this was not the subject of investigation in the current study. Furthermore, nanoparticle synthesis was carried out under experimental conditions where the diblock copolymer chains are self-organized in the form of spherical micelles (i.e., at $\mathrm{pH}>\mathrm{p} K_{\mathrm{a}}$ in the case of $\mathrm{pH}$-responsive systems).

The synthesis was studied by UV-Vis spectroscopy, and the results followed the typical behavior already reported in all of the cases. ${ }^{9,33}$ Briefly, an absorption band at around $300 \mathrm{~nm}$ due to the presence of $\mathrm{Pd}^{\mathrm{II}}$ ions in solution is observed before the addition of $\mathrm{NaBH}_{4} \cdot{ }^{5,33-35}$ This signal disappears after $\mathrm{Pd}^{\mathrm{II}}$ ions are quantitatively reduced to $\mathrm{Pd}^{0}$ upon addition of $\mathrm{NaBH}_{4}$. The resulting PdNP also absorb light to a certain extent within the whole range of $300-800 \mathrm{~nm}$, and their maximum absorbance is at a higher wavelength (data not shown).

The size of metallic nanoparticles used in this study was $7.0 \pm 5.5 \mathrm{~nm}$ as measured by TEM imaging (see TEM micrograph in Figure 1a and corresponding particle size distribution in Figure 1b). Their arrangement/stabilization

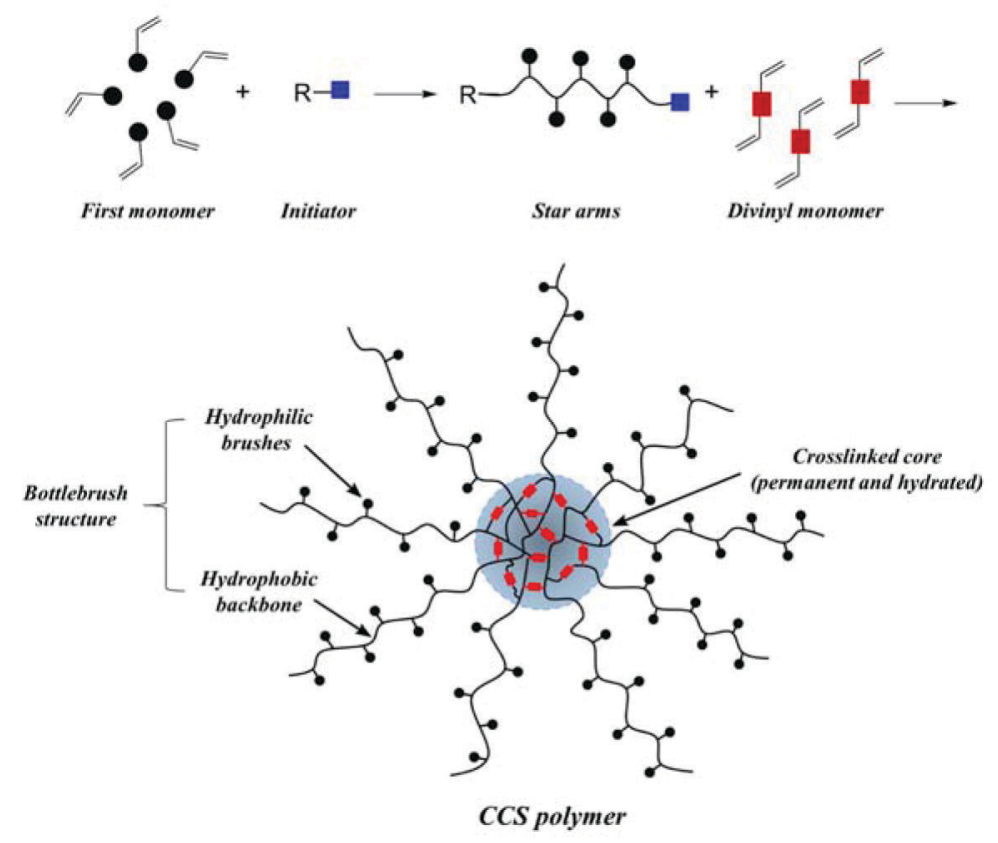

Scheme 1. Envisioned approach to synthesizing nanogels through a one-pot procedure comprising arm-first approach with sequential addition of monomers employing the simple and versatile activators regenerated by electron transfer atom transfer radical polymerization (ARGET ATRP) technique. 
by the capping agent was dependent on the macromolecular architecture, and it should be noted that there is no constraints for the metallic particle location. It can grow within the core, at the core/shell interface, in the shell region, or even find stabilizing molecules after seeding/ growing in the solution bulk. Eventually, the capping agent molecules stabilize reactive metallic particles. In general, the main population of hybrid particles had a number-averaged radius of ca. $25 \mathrm{~nm}$, as revealed by DLS (Figure 1c). A slow relaxation mode corresponding larger particles was consistently evident for these systems due to dynamic intermolecular interaction and aggregation. The dynamic nature of these scattering objects can be inferred from the fact that filtration with $100 \mathrm{~nm}$ pore size filters practically does not affect the distribution of relaxation times. The structure of the capping layer is discussed in more detail below along with catalytic assays.

\section{Catalytic hydrogenation of Nip}

Previously, we found that the most active catalysts in the $p$-nitrophenol (Nip) reaction are also effective in the SuzukiMiyaura cross-coupling reaction between 4-iodoanisole and phenylboronic acid. Indeed, the hydrogenation of Nip has become a tool widely used to evaluate the catalytic activity of nanoparticle systems, primarily because the reaction progress can easily be followed in situ by UV-Vis spectroscopy. Further details can be found elsewhere.9,29

Table 2 shows the observed rate constant $\left(\mathrm{k}_{\mathrm{obs}}\right)$ for the reduction of Nip to Amp catalyzed by polymer@PdNPs. The catalytic activity of polymer@PdNPs evaluated on the basis of $k_{\text {obs }}$ values revealed the capping agent effect. First, we considered homopolymer $\mathbf{1}$ in this study not only because it is largely used in organic chemistry laboratories to prepare PdNP for Suzuki coupling reactions, ${ }^{5,34,36,37}$ but also because we wanted to compare it with the structurally related counterpart 2 . The former is a linear chain of ethylene oxide repeat units, and the latter is a bottlebrush polymer with a hydrophobic methacrylate backbone and short side chains made of ethylene oxide repeat units. The hydrophilic monomer is the same, but it is distributed differently along the chain. These two polymers at the same concentration (very low in this work) provide distinct stability to the particulate systems, with aggregation normally occurring after a few hours in case of $\mathbf{1}$ (appearance of a black solid precipitate). The $\mathrm{k}_{\mathrm{obs}}$ values in Table 2 showed that catalytic activity of $2 @$ PdNPs $\left(\mathrm{k}_{\mathrm{obs}}=3.5 \times 10^{-2} \mathrm{~s}^{-1}\right)$ is higher than $1 @$ PdNPs $\left(\mathrm{k}_{\mathrm{obs}}=2.7 \times 10^{-2} \mathrm{~s}^{-1}\right)$, which we attribute to a better hydrophilic/hydrophobic balance in 2@PdNP that favors diffusion of reactants and products. Recently, $\mathrm{Niu}$ and $\mathrm{Li}^{38}$ noted that the exact way in which different
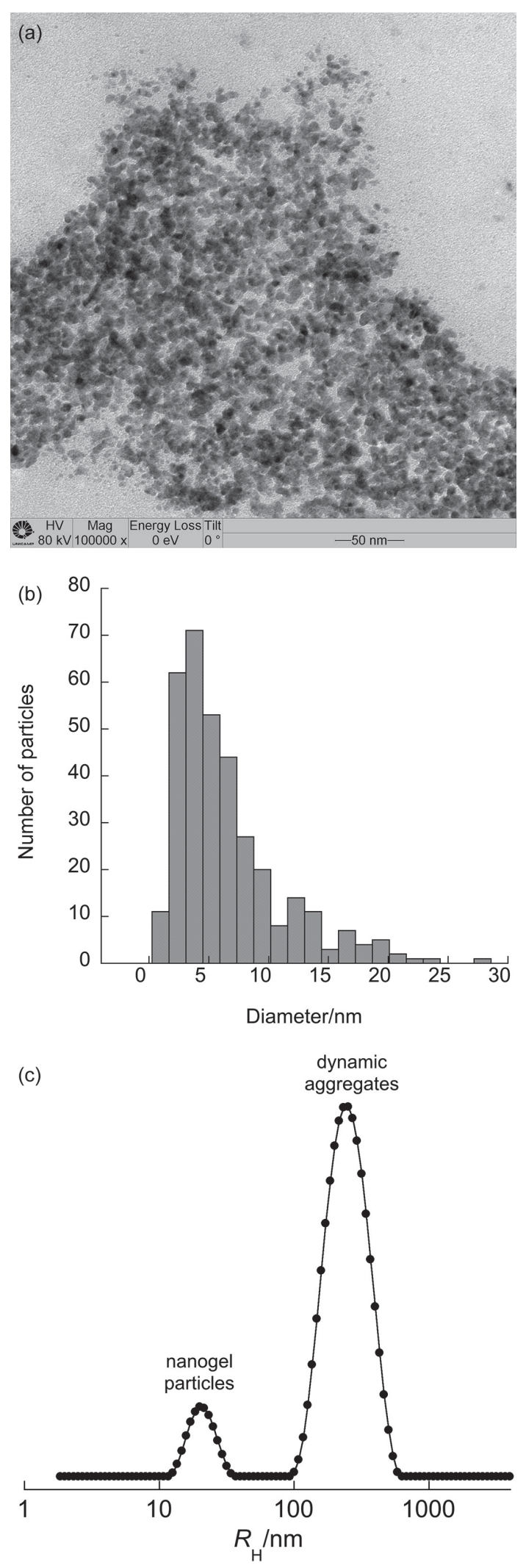

Figure 1. (a) TEM micrograph and (b) corresponding palladium metallic particle size distribution by image analysis; (c) DLS intensity-averaged distribution of the relaxation times obtained by CONTIN analysis of autocorrelation functions measured at scattering angle of $90^{\circ}$ for 6@PdNP hybrid particles used in this study. 
Table 2. PdNP hydrodynamic radius and observed rate constant $\left(\mathrm{k}_{\mathrm{obs}}\right)$ for the reduction of Nip to Amp

\begin{tabular}{|c|c|c|}
\hline Capping agent & $\mathrm{R}_{\mathrm{H}}^{\mathrm{a}} / \mathrm{nm}$ & Rate constant $\left(\mathrm{k}_{\mathrm{obs}}\right) \times 10^{2} / \mathrm{s}^{-1}$ \\
\hline $1-\mathrm{PEO}_{113}$ & $112^{\mathrm{b}}$ & $2.700 \pm 0.002$ \\
\hline 2 - PPOEGMA 67 & $43^{\mathrm{b}}$ & $3.536 \pm 0.001$ \\
\hline $3-\mathrm{PEO}_{113}-b-\mathrm{PDEA}_{50}$ & 42 & $2.980 \pm 0.771$ \\
\hline 4 - PPOEGMA $_{45}-b-$ PDEA $_{80}$ & $14^{\mathrm{b}}$ & $0.812 \pm 0.002$ \\
\hline 5 - PPOEGMA $60-b$-P(POEGMA-co-EGDMA 2$)$ & $25^{\mathrm{b}}$ & $10.031 \pm 0.009$ \\
\hline 6 - PPOEGMA ${ }_{60}-b$-P(POEGMA-co-EGDMA-co-DEA) & $29^{b}$ & $2.500 \pm 0.010$ \\
\hline
\end{tabular}

aHydrodynamic radius of polymer@PdNPs determined by dynamic light scattering (DLS) $24 \mathrm{~h}$ after the synthesis; ba slow relaxation mode corresponding to $\mathrm{R}_{\mathrm{H}}=100-200 \mathrm{~nm}$ is also evident for these systems due to dynamic intermolecular interaction and aggregation. PEO: poly(ethylene oxide); PPOEGMA: poly[poly(ethylene glycol) methyl ether methacrylate]; PDEA: poly[2-(diethylamino)ethyl methacrylate]; POEGMA: poly(ethylene glycol) methyl ether methacrylate; EGDMA: ethylene glycol dimethacrylate; DEA: 2-(diethylamino)ethyl methacrylate.

conformations influence the access of reactants to a catalyst still remains unclear, but the capping layer is certainly detrimental to this process.

It is worth noting that the activity of $\mathbf{2} @$ PdNPs is already higher than all of the block copolymers investigated in an early study, ${ }^{9}$ and one order of magnitude higher than those previously observed for PdNPs prepared in the presence of poly(amidoamine) and poly(propyleneimine) dendrimers, ${ }^{39}$ and poly(ethylene oxide) functionalized with triazoyl rings. ${ }^{40}$ Even in the best possible scenario, the synthesis of any block copolymer would obviously be more complicated than the synthesis of $\mathbf{2}$, which is synthesized through a one-pot, fast reaction in alcoholic medium using all commercially available reactants and ppm amounts of metals (copper).

The presence of amino-groups in the capping agent is clearly not appealing for catalyst systems. Upon chain extension of $\mathbf{2}$ with DEA monomer to produce $\mathbf{4}$, a linear diblock copolymer that self-assembles into spherical core-shell micelles, $\mathrm{k}_{\mathrm{obs}}$ decreased from $3.5 \times 10^{-2}$ down to $0.8 \times 10^{-2} \mathrm{~s}^{-1}$. Under the experimental conditions of Nip reduction ( $\mathrm{pH}=7.4), 80 \%$ of the nitrogen atoms in DEA are deprotonated, and thus form a rather hydrophobic microphase, which ultimately hinders both the access of reactants to catalytic sites and the diffusion of products out of the particles surface. The same rationale also applies for $3 @$ PdNPs.

The CCS polymer (nanogel) 5 yielded an exceedingly effective catalyst system. The characteristic $\mathrm{k}_{\mathrm{obs}}$ value for $5 @$ PdNPs reached an impressive $10.0 \times 10^{-2} \mathrm{~s}^{-1}$, being threefold higher than any other case considered in our recent work. This nanogel is a $25 \mathrm{~nm}$ highly hydrated unimolecular single object with a water-soluble PPOEGMA outer shell and a swollen core of the same material. The adequate hydrophilic/hydrophobic balance of PPOEGMA in regard to Nip/Amp (discussed above) is enhanced in the nanogel structure, and the substrate can then pre-concentrate closer to the metallic nanoparticle. As a result, the local high concentration increases the overall reaction rate.
In a recent study, da Costa et al. ${ }^{19}$ working on a $\mathrm{P}(N, N$-diethylacrylamide $)-b-\mathrm{P}(N, N$-diethylacrylamideco- $N, N$-dimethylaminoethyl acrylate-co- $N, N$-methylenebis (acrylamide) core-shell nanogel, reported extensive Pd leaching during catalysis. According to the authors, the leaching process begins after the oxidation step, which is believed to take place in the nanogel core. The polymer@PdNPs sol is stable overtime and PdNPs remain stable within the nanogel up to the moment it enters a catalytic cycle. The same phenomenon is also expected in the present case, especially owing to the swollen nature of the core. With active Pd species outside the polymeric particle, substrate access to the metallic center is faster, and so is the reaction.

In the light of this result, we designed the nanogel $\mathbf{6}$ by incorporating a small fraction of DEA in the core composition. The reasoning behind this idea is that such a hydrophobic monomer would generate a compact microphase, and would thus create a physical barrier against leaching. The problem with this strategy is that, besides preventing Pd leaching, the presence of DEA also generates a diffusion barrier for reactants and products, as already discussed above. Indeed, the high activity $5 @$ PdNPs was lost as judged from the decrease of $k_{\text {obs }}$ down to $2.5 \times 10^{-2} \mathrm{~s}^{-1}$ for $\mathbf{6} @ \mathrm{PdNPs}$. We also believe it is reasonable to consider, in the present case, that palladium nanoparticles may be slightly poisoned by electron donor nitrogen atoms of amino groups, although the extent of poisoning is not comparable to pyridyl moieties which can scavenge palladium species.

\section{Catalyst recycling}

The concept of catalyst recycling involves the recovery of the catalyst for subsequent re-use in any other catalytic process. It is obviously a very exciting prospect for developing sustainable chemical processes. In the present case, polymer@PdNPs can be separated as a grayish/dark 
gum from the reaction medium by ultracentrifugation at $12,000 \mathrm{rpm}$ at $5{ }^{\circ} \mathrm{C}$ for $25 \mathrm{~min}$. Under these conditions, particles as small as polymeric micelles deposit as a sediment almost quantitatively, as attested by light scattering experiments. The total scattered light intensity of the supernatant (solvent) was close to pure water after centrifugation (Figure 2a), and the deposit (particles) could be re-dispersed in water within ca. 5 min with ultrasound assistance. The recycled materials sustained activity after recycling in Nip reduction reactions, apart from 5@PdNPs system, which lost activity in situ upon successive additions (see below).

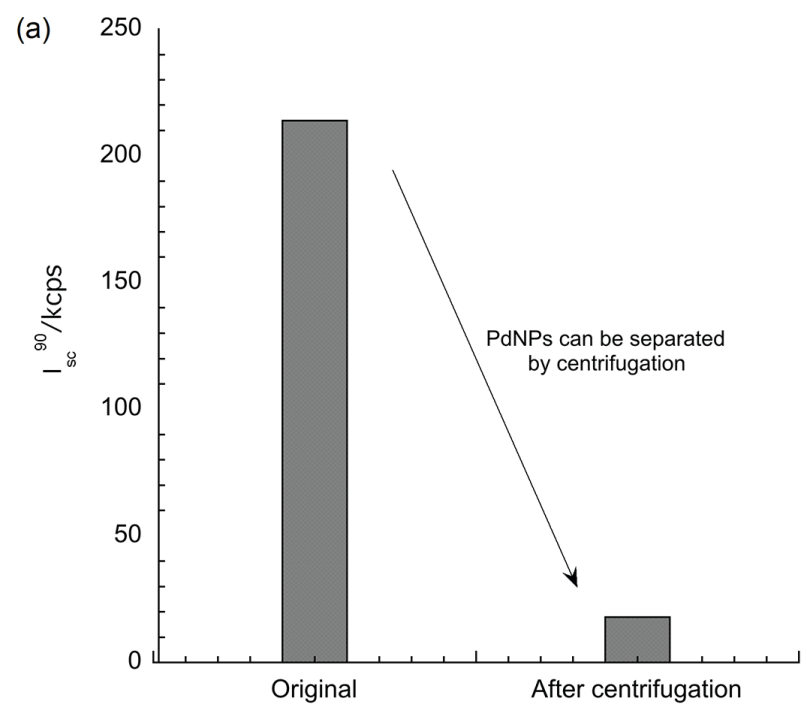

Nanoparticle solution

(b)

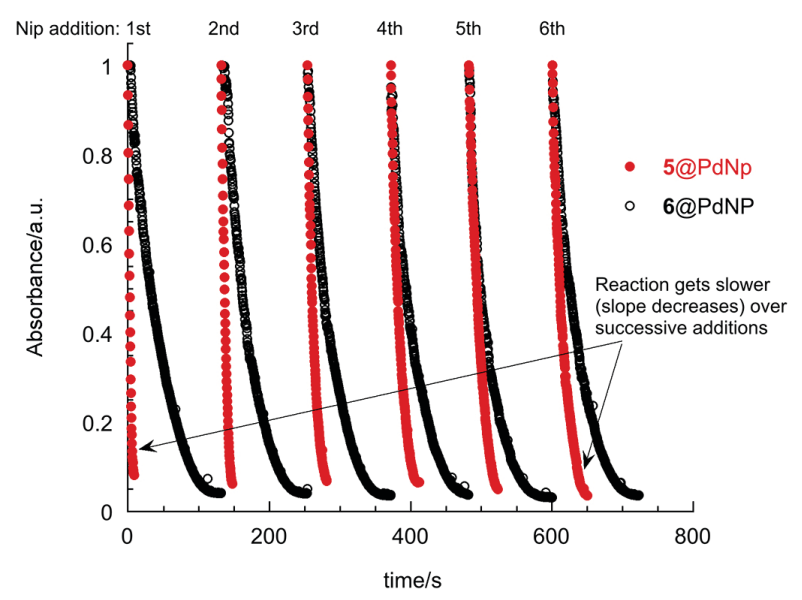

Figure 2. (a) Total scattered light intensity of a $6 @$ PdNPs solution before and after centrifugation at $12,000 \mathrm{rpm}$ at $5{ }^{\circ} \mathrm{C}$ during $25 \mathrm{~min}$, showing efficient particle separation; (b) dilution-corrected absorbance variation at $400 \mathrm{~nm}$ recorded as a function of time during the Nip reduction reaction at $25{ }^{\circ} \mathrm{C}$ in the presence of $\mathbf{5} @ \mathrm{PdNPs}$ and $\mathbf{6} @ \mathrm{PdNPs}$ after successive additions of the substrate $(6$ aliquots of Nip were added after the initial reaction $)\left([\mathrm{Nip}]_{\text {initial }}=1.0 \times 10^{-4} \mathrm{~mol} \mathrm{~L}^{-1},\left[\mathrm{NaBH}_{4}\right]=5.0 \times 10^{-3} \mathrm{~mol} \mathrm{~L}^{-1}\right.$, $\left.[\mathrm{NaOH}]=1.0 \times 10^{-3} \mathrm{~mol} \mathrm{~L}-1 ;[\mathrm{Pd}]=2.5 \times 10^{-5} \mathrm{~mol} \mathrm{~L}^{-1}\right)$.
We were also interested in evaluating whether the particles remain active after an initial batch reaction. Two nanogel systems were submitted to this test, namely 5@PdNPs and 6@PdNps. Figure 2b shows that Nip is promptly reduced to Amp after successive additions provided that a high molar excess of the reducing agent $\mathrm{NaBH}_{4}$ is present in the medium. At first, slopes of each curve reflected the difference in terms of $\mathrm{k}_{\mathrm{obs}}$, with $\mathbf{5} @ \mathrm{PdNP}$ leading to faster reactions than $6 @ \mathrm{PdNp}$. It is worth noting, however, the difference in terms of stability between these two systems. The $6 @$ PdNp catalyst yielded constant slopes after successive additions, but 5@PdNp progressively lost its activity as seen from the decrease in the slopes of absorbance vs. time curves (arrows indication in Figure 2b). This was attributed to the phenomenon already described above.

\section{Suzuki coupling reactions}

Suzuki cross-coupling of organoboronic acids with aryl halides to produce biaryls has contributed enormously to sustainable development of chemical industry where $\mathrm{C}-\mathrm{C}$ bond formation is necessary. ${ }^{41,42}$ The polymer@PdNPs considered in this study were evaluated as catalysts in a typical Suzuki reaction between phenylboronic acid and 4-iodoanisole (Scheme 2). In a previous correlate study by our group, ${ }^{9}$ the well-known effect of carbon-halogen bond dissociation energies was evident, as expected, with aryl iodides being more reactive than other aryl halides. ${ }^{1,41}$ In the present case, we elected to restrict the analysis to 4-iodoanisole. ${ }^{1} \mathrm{H}$ NMR spectroscopy experiments confirmed the identity and purity of the expected product 4-methoxybiphenyl (see Figure 3), and therefore the efficiency of the synthetic method.

Table 3 shows the results of Suzuki coupling reactions carried out in presence of catalysts developed in this study. Initially, the effect of capping agent was evaluated (Table 3, entries 1-6). Aggregation was observed for $1 @$ PdNPs system during the reaction, and it reflected on the low yield of biaryl product. Although PEO (poly(ethylene oxide)) has been often used for this same purpose with satisfactory results, ${ }^{5}$ significantly less capping agent is applied here ( $1 / 5$ of the amount in some cases). All other catalysts were, in general, active in mediating the reaction towards the formation of the expected product, in almost quantitative yields, under relatively mild conditions, after only $4 \mathrm{~h}$ (Table 3 , entries 2-6). In spite of the low capping agent concentration, nanoparticles remained stable during the reaction and performed similarly to those previously used by other authors. ${ }^{35-37,42,43}$ This result indeed proved one of the hypothesis of our previous and current studies: 


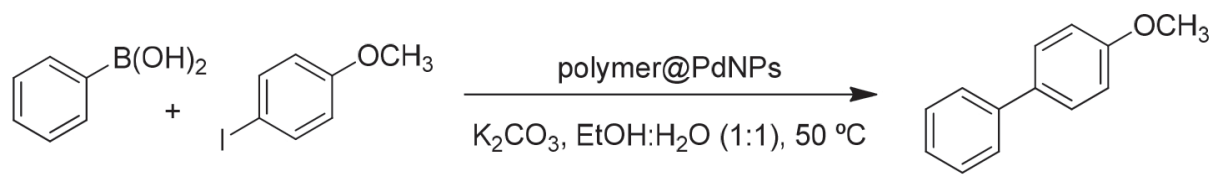

Scheme 2. Suzuki coupling reaction between phenylboronic acid and 4-iodoanisole used to test the catalytic activity of the selected polymer@PdNPs.

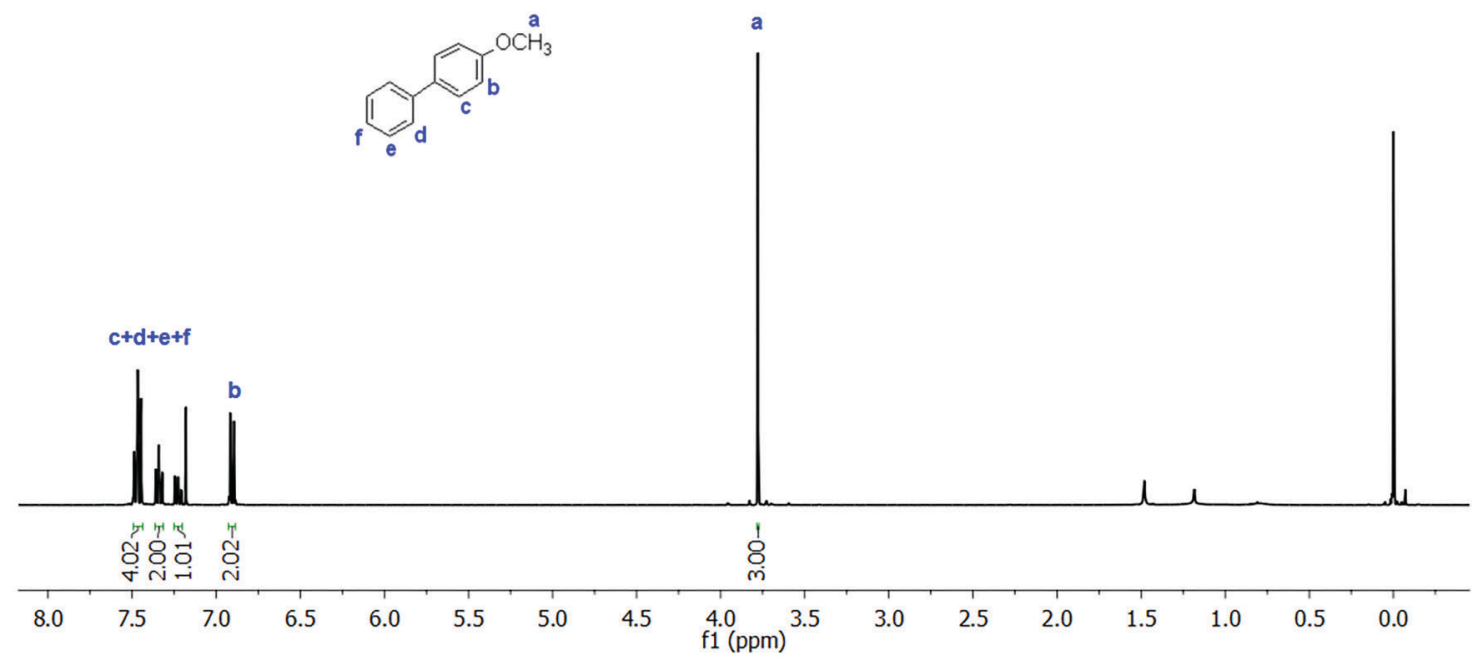

Figure 3. Assigned ${ }^{1} \mathrm{H}$ NMR spectrum ( $400 \mathrm{MHz}, \mathrm{CDCl}_{3}$ ) of 4-methoxybiphenyl product resulting from Suzuki coupling reaction between phenylboronic acid and 4-iodoanisole.

precise macromolecular engineering allows for better design of capping agents.

In the sequence, we investigated the effect of catalyst concentration for $2 @$ PdNPs (a bottlebrush polymer; Table 3, entries 7-11) and 5@PdNPs (a core-crosslinked star polymer nanogel with bottlebrush arms; Table 3, entries 12-16). Reactions carried out in presence of $5 @ P d N P s$ catalyst system yielded near quantitative conversions for concentrations as low as $1 \mathrm{~mol} \% \mathrm{Pd}$. At this metal content, the simpler structure of $\mathbf{2} @$ PdNPs was not as effective, but still showed a remarkable activity (yield $>85 \%$, Table 3, entry 9). Below 1.0 mol\% Pd 5@PdNPs, the coupling reaction was not complete within $4 \mathrm{~h}$ at $50{ }^{\circ} \mathrm{C}$ in any case. It is clear that $1.0 \mathrm{~mol} \% \mathrm{Pd}$ is a very low threshold as compared to loadings most often used in the field for particulate systems. ${ }^{1}$ Besides, it is imperative to take into account the mild conditions employed in this work. Suzuki couplings were conducted at $50^{\circ} \mathrm{C}$, and also for this reason a $4 \mathrm{~h}$ period was required to achieve an appropriate product yield (Table 3, entries 17-20). Most often, such a kind of reaction is performed at $80{ }^{\circ} \mathrm{C}$.

Turn over frequency (TOF) has often been used to characterize catalytic activities. However, such a parameter has been put into discussion in case of complex particulate systems due to uncertainties related not only to the number of active sites, but also to different activity of several surface positions, as addressed by Kozuch and Martin ${ }^{44}$ in detail. In the current study, TOF values have been calculated using the method described for a similar case by Zhang et al. ${ }^{45}$ in which only atoms at the particle surface are considered to be active sites. The particle size parameter was taken as the size average determined by image analysis (as illustrated in Figure $1 \mathrm{~b}$ and corresponding discussion), even though there is certain dispersion. The TOF values shown in Table 3 confirmed the catalyst activity trend already described above. The values cannot be directly compared to those reported in the literature ${ }^{10,11}$ because the reactions were carried out under mild conditions in this study.

Overall, the results shown in Table 3 showed the clear advantage of the current approach: the easy access to the catalyst systems represented by the possibility of going from monomers to ready-to-use catalysts in only two-steps. In addition, significantly less capping agent is applied ( $1 / 5$ of the amount in some cases) to the synthesis of polymer@PdNPs, which, in spite of such a fact, remained stable during the reaction and performed similarly to those previously used by other authors. ${ }^{35-37,42,43}$ It is, therefore, a step forward in terms of environmental and economic prospects.

Polymer@PdNPs sustain activity after recycling in Suzuki coupling reactions, as they do in Nip reductions. However, the catalyst recovery from the reaction medium is more complicated in the case of Suzuki type reactions due to the reaction workup that is necessary to separate the 
Table 3. Results of Suzuki coupling reaction between phenylboronic acid and iodoanisole using polymer@PdNPs as the catalyst ${ }^{\mathrm{a}}$

\begin{tabular}{|c|c|c|c|c|c|}
\hline entry & Capping agent & $\mathrm{Pd} / \mathrm{mol} \%$ & time $/ \mathrm{h}$ & Yield $^{\mathrm{b}} / \%$ & $\mathrm{TOF}^{\mathrm{c}} / \mathrm{h}^{-1}$ \\
\hline \multicolumn{6}{|c|}{ Effect of capping agent } \\
\hline 1 & $1-\mathrm{PEO}_{113}$ & 10 & 4 & 77 & 12.0 \\
\hline 2 & 2 - PPOEGMA 67 & 10 & 4 & 97 & 15.2 \\
\hline 3 & $3-\mathrm{PEO}_{113}-b-\mathrm{PDEA}_{50}$ & 10 & 4 & 96 & 15.0 \\
\hline 4 & 4 - PDEA $_{80}-b$-PPOEGMA 45 & 10 & 4 & 89 & 13.0 \\
\hline 5 & 5 - PPOEGMA $_{60}-b-\mathrm{P}\left(\mathrm{POEGMA}-c o-\mathrm{EGDMA}_{2}\right)$ & 10 & 4 & 98 & 15.3 \\
\hline 6 & 6 - PPOEGMA $_{60}-b$-P(POEGMA-co-EGDMA-co-DEA) & 10 & 4 & 97 & 15.2 \\
\hline \multicolumn{6}{|c|}{ Effect of catalyst concentration } \\
\hline 7 & 2 - PPOEGMA $_{67}$ & 10 & 4 & 97 & 15.2 \\
\hline 8 & 2 - PPOEGMA $_{67}$ & 5 & 4 & 89 & 27.8 \\
\hline 9 & 2 - PPOEGMA $_{67}$ & 1 & 4 & 88 & 137.5 \\
\hline 10 & 2 - PPOEGMA $_{67}$ & 0.5 & 4 & 75 & 234.8 \\
\hline 11 & 2 - PPOEGMA $_{67}$ & 0.1 & 4 & 24 & 375.0 \\
\hline 12 & 5 - PPOEGMA $_{60}-b-\mathrm{P}\left(\mathrm{POEGMA}-c o-\mathrm{EGDMA}_{2}\right)$ & 10 & 4 & 98 & 15.3 \\
\hline 13 & 5 - PPOEGMA $_{60}-b$-P(POEGMA-co-EGDMA 2$)$ & 5 & 4 & 99 & 30.9 \\
\hline 14 & 5 - PPOEGMA $_{60}-b-\mathrm{P}\left(\mathrm{POEGMA}-c o-\mathrm{EGDMA}_{2}\right)$ & 1 & 4 & 97 & 151.5 \\
\hline 15 & 5 - PPOEGMA $_{60}-b$-P(POEGMA-co-EGDMA 2$)$ & 0.5 & 4 & 85 & 265.6 \\
\hline 16 & 5 - PPOEGMA $_{60}-b$-P(POEGMA-co-EGDMA 2$)$ & 0.1 & 4 & 17 & 265.6 \\
\hline \multicolumn{6}{|c|}{ Effect of reaction time } \\
\hline 17 & 5 - PPOEGMA $_{60}-b-\mathrm{P}\left(\mathrm{POEGMA}-c o-\mathrm{EGDMA}_{2}\right)$ & 1 & 4 & 97 & 151.5 \\
\hline 18 & 5 - PPOEGMA $_{60}-b-\mathrm{P}\left(\mathrm{POEGMA}-c o-\mathrm{EGDMA}_{2}\right)$ & 1 & 3.3 & 93 & 176.1 \\
\hline 19 & 5 - PPOEGMA ${ }_{60}-b-\mathrm{P}\left(\mathrm{POEGMA-co-EGDMA}{ }_{2}\right)$ & 1 & 2.7 & 87 & 201.4 \\
\hline 20 & 5 - PPOEGMA ${ }_{60}-b$-P(POEGMA-co-EGDMA 2$)$ & 1 & 2 & 80 & 250.0 \\
\hline
\end{tabular}

a Reaction conditions: $\mathrm{PhB}(\mathrm{OH})_{2}(0.38 \mathrm{mmol}), \mathrm{K}_{2} \mathrm{CO}_{3}(0.50 \mathrm{mmol}), \mathrm{EtOH}: \mathrm{H}_{2} \mathrm{O}(0.80 \mathrm{~mL}), \mathrm{IC}_{6} \mathrm{H}_{4} \mathrm{OCH}_{3}(0.25 \mathrm{mmol})$; bisolated yield; cturn over frequency defined as number of mol of substrate transformed per mol of active catalyst at the particle surface per hour. PEO: poly(ethylene oxide); PPOEGMA: poly[poly(ethylene glycol) methyl ether methacrylate]; PDEA: poly[2-(diethylamino)ethyl methacrylate]; POEGMA: poly(ethylene glycol) methyl ether methacrylate; EGDMA: ethylene glycol dimethacrylate; DEA: 2-(diethylamino)ethyl methacrylate.

desired product. Considering this fact, we decided to test the possibility of catalyst recycling by applying the so-called "dip catalyst" strategy. ${ }^{46-49}$ The dip catalyst preparation was carried out following a procedure adapted from the recent work of Zheng et al., ${ }^{49}$ who used cellulose paper as substrate. In our study, the composite catalytic system was prepared by immersion of cellulose filter paper pieces $(15 \times 15 \mathrm{~mm})$ in $6 @$ PdNPs solutions (prepared as described in the Nanoparticle synthesis section), followed by vacuum drying for at least $24 \mathrm{~h}$. This process was repeated three times. Strong van der Waals forces and hydrophobic interactions between nanogel structure and cellulose lead to catalyst immobilization. The resulting composite dip catalyst system was then used in three successive Suzuki coupling reactions. After each reaction, the small piece of paper was simply fetched and transferred to another vessel to catalyze the subsequent reaction. At this point in time, we can only confirm the near quantitative conversion of iodoanisole reactant into the expected reaction product in the three successive reactions, as judged from thin layer chromatography studies. We are currently working on further characterization of the dip catalyst (particle loading and stability) and reaction features. These results already confirm that polymer@PdNPs can be recycled and sustain activity, in agreement with earlier studies that have systematically corroborated the feasibility of PdNPs recycling. ${ }^{2,46,49}$

\section{Conclusions}

Nanogels consisting of water-soluble building blocks are suitable capping agents for palladium nanoparticle catalyst systems because, as highly hydrated unimolecular single objects, they practically do not hinder diffusion of reactants and products through the nanoreactor shell while still providing good particle stability. In spite the fact that nanogels have a complex structure, the current understating of macromolecular chemistry allows easy access to them 
by straight-forward methods, as shown in this study. The ARGET ATRP polymerization technique with sequential addition of monomers uses only commercially available reactants, and can be applied to the synthesis of polymer nanogels in one-pot reactions. The quantitative monomer conversion makes the final product ready to use (without any further purification) for palladium nanoparticle synthesis in a subsequent step. When tested in $p$-nitrophenol reduction and Suzuki cross-coupling reactions, these systems performed similarly to those that required more laborious reaction workups by polymer chemist specialists.

Despite the low concentration of stabilizer used, compared to previous studies, the catalysts can still be recycled (recovered/re-used) using standard protocols applied in this field.

\section{Acknowledgments}

The authors acknowledge financial support from CNPq, CAPES and FAPERGS. We also thank A. G. O. de Freitas, P. I. R. Muraro, J. Vargas, and M. C. Gonçalves for their assistance on experimental procedures.

\section{References}

1. Yin, L.; Liebscher, J.; Chem. Rev. 2007, 107, 133.

2. Pérez-Lorenzo, M.; J. Phys. Chem. Lett. 2012, 3, 167.

3. Ikegami, S.; Hamamoto, H.; Chem. Rev. 2009, 109, 583.

4. Signori, A. M.; Santos, K. O.; Eising, R.; Albuquerque, B. L.; Giacomelli, F. C.; Domingos, J. B.; Langmuir 2010, 26, 17772.

5. Harraz, F. A.; El-Hout, S. E.; Killa, H. M.; Ibrahim, I. A.; J. Catal. 2012, 286, 184.

6. Sun, Q.; Zhang, X.-Q.; Wang, Y.; Lu, A.-H.; Chin. J. Catal. 2015, 36, 683 .

7. Cai, S.; Wang, D.; Niu, Z.; Li, Y.; Chin. J. Catal. 2013, 34, 1964.

8. Muller, A. H. E.; Matyjaszewski, K.; Controlled and Living Polymerizations: From Mechanisms to Applications; WileyVCH: Weinheim, 2009.

9. Bortolotto, T.; Facchinetto, S. E.; Trindade, S. G.; Ossig, A.; Petzhold, C. L.; Vargas, J.; Rodrigues, O. E. D.; Giacomelli, C.; Schmidt, V.; J. Colloid Interface Sci. 2015, 439, 154.

10. Chen, Z.; Liang, Y.; Jia, D.-S.; Cui, Z.-M.; Song, W.-G.; Chin. J. Catal. 2017, 38, 651.

11. Fan, H.; Qi, Z.; Sui, D.; Mao, F.; Chen, R.; Huang, J.; Chin. J. Catal. 2017, 38, 589.

12. Chen, G.; Wang, F.; Wang, Y.; Zhang, X.; Qin, H.; Zou, H.; Xu, J.; Chin. J. Catal. 2014, 35, 540.

13. Zhang, C.; Miao, M.; Cao, X.; An, Z.; Polym. Chem. 2012, 3, 2656.

14. Chen, Q.; Cao, X.; Xu, Y.; An, Z.; Macromol. Rapid Commun. 2013, 34, 1507.
15. Kuckling, D.; Wycisk, A.; J. Polym. Sci., Part A: Polym. Chem. 2013, 51, 2980.

16. Cao, X.; Zhang, C.; Wu, S.; An, Z.; Polym. Chem. 2014, 5, 4277.

17. Seto, H.; Morii, T.; Yoneda, T.; Murakami, T.; Hoshino, Y.; Miura, Y.; Chem. Lett. 2013, 42, 301.

18. Seto, H.; Yoneda, T.; Morii, T.; Hoshino, Y.; Miura, Y.; Murakami, T.; AIChE J. 2015, 61, 582.

19. da Costa, A. P.; Nunes, D. R.; Tharaud, M.; Oble, J.; Poli, G.; Rieger, J.; ChemCatChem 2017, 9, 2167.

20. Matyjaszewski, K.; Macromolecules 2012, 45, 4015.

21. Simakova, A.; Averick, S. E.; Konkolewicz, D.; Matyjaszewski, K.; Macromolecules 2012, 45, 6371.

22. Liu, S.; Weaver, J. V. M.; Tang, Y.; Billingham, N. C.; Armes, S. P.; Tribe, K.; Macromolecules 2002, 35, 6121.

23. Giacomelli, C.; Schmidt, V.; Borsali, R.; Langmuir 2007, 23, 6947.

24. Jakubowski, W.; Matyjaszewski, K.; Angew. Chem., Int. Ed. 2006, 45, 4482.

25. Jakubowski, W.; Min, K.; Matyjaszewski, K.; Macromolecules 2006, 39, 39.

26. Mueller, L.; Matyjaszewski, K.; Macromol. React. Eng. 2010, 4, 180.

27. Wunder, S.; Lu, Y.; Albrecht, M.; Ballauff, M.; ACS Catal. 2011, $1,908$.

28. Wunder, S.; Polzer, F.; Lu, Y.; Mei, Y.; Ballauff, M.; J. Phys. Chem. C 2010, 114, 8814.

29. Mei, Y.; Lu, Y.; Polzer, F.; Ballauff, M.; Drechsler, M.; Chem. Mater. 2007, 19, 1062.

30. Dong, H.; Matyjaszewski, K.; Macromolecules 2008, 41, 6868.

31. Burdyńska, J.; Cho, H. Y.; Mueller, L.; Matyjaszewski, K.; Macromolecules 2010, 43, 9227.

32. Mendonça, P. V.; Averick, S. E.; Konkolewicz, D.; Serra, A. C.; Popov, A. V.; Guliashvili, T.; Matyjaszewski, K.; Coelho, J. F. J.; Macromolecules 2014, 47, 4615.

33. Chen, C.-W.; Akashi, M.; Langmuir 1997, 13, 6465.

34. Luo, C.; Zhang, Y.; Wang, Y.; J. Mol. Catal. A: Chem. 2005 , $229,7$.

35. Cotugno, P.; Monopoli, A.; Ciminale, F.; Cioffi, N.; Nacci, A.; Org. Biomol. Chem. 2012, 10, 808.

36. Han, W.; Liu, C.; Jin, Z.-L.; Org. Lett. 2007, 9, 4005.

37. Du, Z.; Zhou, W.; Wang, F.; Wang, J.-X.; Tetrahedron 2011, 67, 4914.

38. Niu, Z.; Li, Y.; Chem. Mater. 2013, 26, 72.

39. Esumi, K.; Isono, R.; Yoshimura, T.; Langmuir 2003, 20, 237.

40. Deraedt, C.; Salmon, L.; Ruiz, J.; Astruc, D.; Adv. Synth. Catal. 2013, 355, 2992.

41. Miyaura, N.; Suzuki, A.; Chem. Rev. 1995, 95, 2457.

42. Li, Y.; Hong, X. M.; Collard, D. M.; El-Sayed, M. A.; Org. Lett. 2000, 2, 2385.

43. He, Y.; Wang, L.; Cai, C.; J. Appl. Polym. Sci. 2011, 121, 286.

44. Kozuch, S.; Martin, J. M. L.; ACS Catal. 2012, 2, 2787. 
45. Zhang, P.; Sui, Y.; Xiao, G.; Wang, Y.; Wang, C.; Liu, B.; Zou, G.; Zou, B.; J. Mater. Chem. A 2013, 1, 1632.

46. Hariprasad, E.; Radhakrishnan, T. P.; ACS Catal. 2012, 2, 1179.

47. Faria, V. W.; Oliveira, D. G. M.; Kurz, M. H. S.; Gonçalves, F. F.; Scheeren, C. W.; Rosa, G. R.; RSC Adv. 2014, 4, 13446.

48. Oliveira, D. G. M.; Alvarenga, G.; Scheeren, C. W.; Rosa, G. R.; Quim. Nova 2014, 37, 1401.
49. Zheng, G.; Kaefer, K.; Mourdikoudis, S.; Polavarapu, L.; Vaz, B.; Cartmell, S. E.; Bouleghlimat, A.; Buurma, N. J.; Yate, L.; de Lera, Á. R.; Liz-Marzán, L. M.; Pastoriza-Santos, I.; PérezJuste, J.; J. Phys. Chem. Lett. 2015, 6, 230.

Submitted: December 7, 2017 Published online: March 20, 2018 